\title{
Nurses should become more involved in haemophilia research
}

\author{
Jenny Bryan
}

\section{Brian O'Mahony, Chief \\ Executive of the Irish \\ Haemophilia Society and \\ President of the European \\ Haemophilia Consortium, tells Jenny Bryan about the special role that nurses play within the haemophilia community}

What was your early experience of haemophilia nursing?

As someone with severe haemophilia B, my first experience of a comprehensive haemophilia care centre was in Dublin in the mid 1970s, and I've been involved with the Irish Haemophilia Society since the early 80 s. In the early days, there was just a haematologist and a nurse and, from the beginning, it was the nurse who played a pivotal role not just in advising patients and families, but in coordinating,

organising and delivering care.

In Ireland, one of these nurses was Margaret King, who encapsulated the importance of the haemophilia nurse with her common-sense advice and support for patients. After she retired, she joined the Irish Haemophilia Society and she became chief counselor and confidant. In those days, Irish men - like British men didn't talk about their emotions and wouldn't normally speak to a counselor. But they'd talk to Margaret.

There were - and still are - a lot of Margarets and they perform a very special role within the haemophilia community.

What makes haemophilia nurses different from other nurses?

Haemophilia nurses build a special relationship with their patients because they are treating them week-in week-out, month after month, year after year. I'm not suggesting that nurses working in other

Jenny Bryan

Email: jlrbryan@btinternet.com

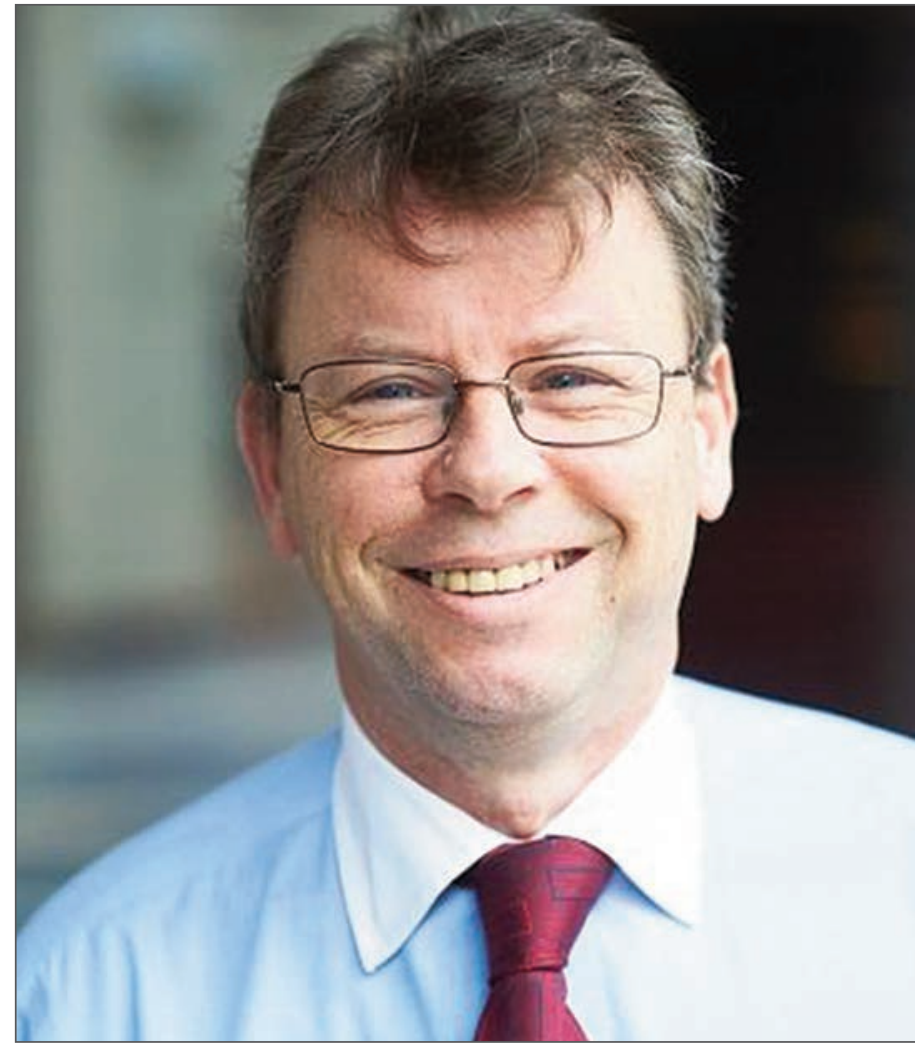

Brian O'Mahony represents the Irish Haemophilia Society on the statutory National Haemophilia Council and as Vice Chairman of the Tender Commission established by the Irish Government. He previously served as Chair of the Irish Haemophilia Society for 17 years and as President of the WFH for 10 years from 1994 to 2004

\section{"Haemophilia nurses are well-placed to carry out surveys on local, regional national and international issues that can help to inform future best practice"}

fields aren't dedicated to their patients, but they don't have the same level of continuing contact with their patients. Haemophilia nurses have developed a unique level of expertise, and many people with haemophilia prefer to talk to the nurse they know really well, than to a junior doctor. One of the attractions of the job is being able to really get to know the condition and the group of patients you are following. It's a long-term commitment.

As past-president of the World Federation of Hemophilia, what differences in haemophilia nursing have you seen worldwide?

So much depends on the status of the nursing profession in a country. In South Africa, where they are highly regarded, nurses are superb at finding and diagnosing people with haemophilia, and they organise outreach services into neighbouring countries and regions, such as Botswana and Lesotho. In other parts of Africa, such as Zimbabwe and Zambia, nurses have very little involvement in decision making. In other countries, for example Vietnam, they play a more supportive role. Here in Ireland, haemophilia nurses have taken on a much bigger role in service development and national policy.

What developments in haemophilia nursing would you like to see in the future? 
I am keen to see nurses become more involved in haemophilia research. We know that haemophilia treatment works but we need more data that clearly demonstrates this fact. We need to show the difference in effects of "on demand" vs prophylactic treatment on joint scores, quality of life and bleeding. We can't do that unless we collect the data, and the obvious people to do that are haemophilia nurses.

I would like to see greater collaboration between nurses both nationally and internationally in assessing treatment regimens and protocols. Everyone has stories about what they have seen working in practice, for example in improving adherence to treatment, and it would be great if these experiences could be shared more widely.

Do haemophilia nurses have time for research?

If you want to get something done, ask a busy person! Nurses already collect a lot of data as part of routine care. When a patient comes in for a routine visit, they could use a simple patient questionnaire to assess quality of life. For example, using an EQ5D, patients score five basic questions - mobility, self-care, usual activities, pain/discomfort and anxiety/depression. It could be completed in 2 minutes and would give valuable information especially if collected methodically at least on an annual basis from each patient.

What other types of research would you like to see?

I think there are some great opportunities to monitor everyday use of Factor VIII or IX and link it with outcomes. When patients use concentrate, they could scan vials with their smart phones, so the information is transmitted to treatment centres. If it became clear that a patient

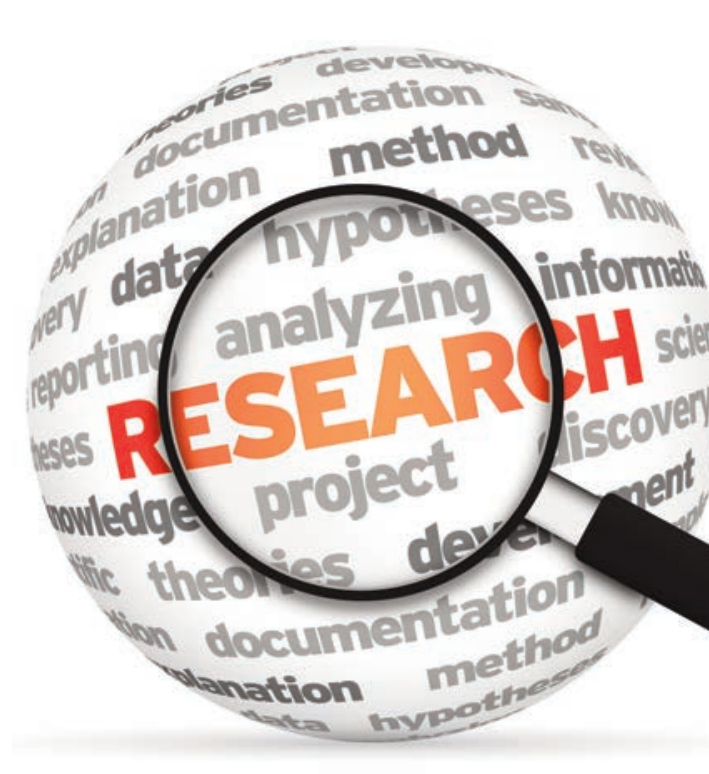

"Everyone has stories about what they have seen working in practice, for example in improving adherence to treatment, and it would be great if these experiences could be shared more widely"

was using more concentrate than expected, a haemophilia nurse could contact them and ask them to come in, or bring forward their review date. If a patient is undertreating, this could be corrected. Use of concentrate could also be linked to days off school or work, not just bleeds. Outcome data from different treatment regimens could be analysed to see if there are any differences over a period of years.

There's also scope for costeffectiveness research, which could help improve clinical management. By looking at the clinical and nursing time needed to monitor treatment or change regimens, we could establish how to save time and achieve the most effective use of concentrates.

There's a treasure trove of information just waiting to be collected and analysed

Where are nurses going to get funding for research?

Research doesn't have to be time consuming and expensive. In 2012, the European Haemophilia Consortium carried out a detailed survey of every aspect of comprehensive care and treatment and organisation of care in 35 European countries. The survey showed a wide range of factor VIII consumption and a significant improvement in access to prophylaxis and home treatment in the 19 countries that had taken part in a previous survey. Two people worked on the results over 6 months, alongside their other work, and the study didn't cost thousands of Euros.

This kind of research can provide valuable insights into real-life practice, and haemophilia nurses are well-placed to carry out surveys on local, regional national and international issues that can help to inform future best practice.

How can nurses raise their profile in haemophilia research?

By doing research and getting published.

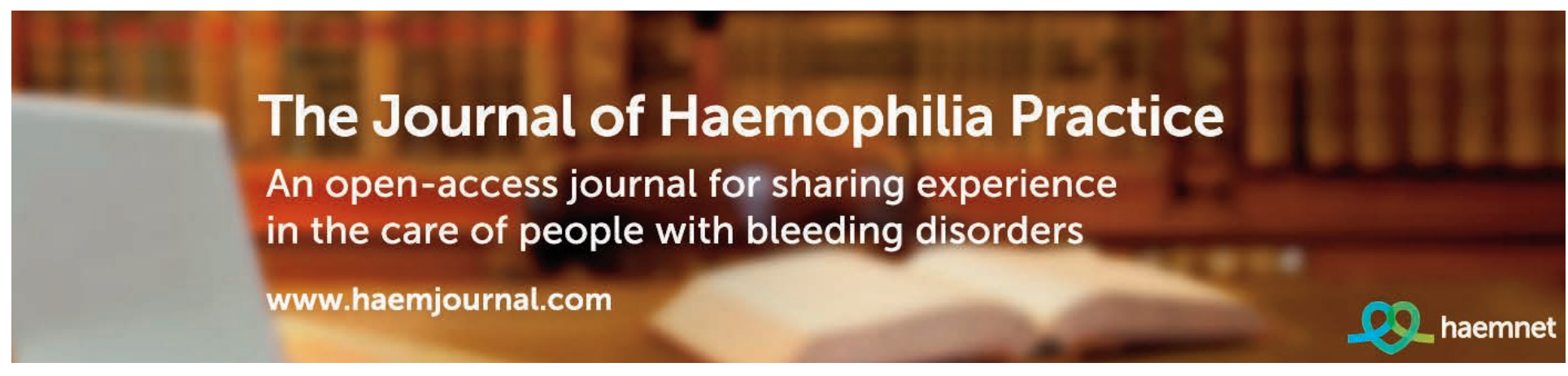

